\title{
Importance of neonatal strain imaging: what are we measuring?
}

\author{
Michal Schäfer ${ }^{1} \cdot$ Richard M. Friesen $^{1} \cdot$ Johannes C. von Alvensleben ${ }^{1}$
}

Published online: 17 May 2021

(c) The Author(s), under exclusive licence to Springer Nature B.V. 2021

Strain imaging derived from speckle-tracking echocardiography (STE) is an increasingly utilized clinical modality for the assessment of bi-ventricular function with important diagnostic, therapeutic, and prognostic roles. Additionally, STE is gaining importance for procedural optimization during cardiac resynchronization therapy. In contrast to ejection fraction, STE derived strain can provide a more comprehensive assessment of global and regional myocardial deformation. More specifically, global longitudinal strain (GLS) is arguably the most sensitive non-invasive measurement of abnormal myocardial performance with the ability to detect subclinical systolic dysfunction in patients with preserved ejection fraction [1]. Consequently, GLS is increasingly gaining attention in pediatric and neonatal echocardiographic studies. A growing body of evidence suggests that GLS and other quantitative myocardial deformation indices collected in neonatal patients have excellent reproducibility and reliability in terms of reader, vendor, test-retest, and even post-processing software variability. The potentially confounding factors of structural heart disease, beat-to-beat variations, arrhythmia, regional wall deformation, and plane of acquisition require additional exploration.

In this issue of the Journal, Khan et al. [2] provide important additional insights and expand our understanding of myocardial deformation imaging. The authors demonstrate that STE derived GLS can be reliably measured in healthy neonates regardless of long-axis plane orientation and drift layer compensation. The typical acquisition plane for left ventricular GLS evaluation is apical 4-chamber or 3 -chamber long axis view. The authors report that there is strong agreement in peak GLS between 4-chamber and 3-plane views consistently throughout the myocardial layers. Expanding upon this, the authors reveal that drift layer compensation yields a statistically significant increase in GLS

Michal Schäfer

michal.schafer@cuanschutz.edu

1 Division of Cardiology, Heart Institute, Children's Hospital Colorado, University of Colorado Denver, Anschutz Medical Campus, 13123 E 16th Ave, Aurora, CO 80045-2560, USA by $0.4 \%$. Drift layer compensation, associated with multiple beat analysis, is primarily influenced by the trough plane motion and global myocardial displacement. Although the finding is statistically significant, its overall effect is arguably negligible considering that both in vitro and in vivo STE validation studies have revealed measurement error for GLS of approximately 1\% [3]. It bears mentioning, however, that beat-to-beat variability and its effect on strain imaging deserves further investigation in children and neonates with arrhythmias as these are inherently associated with variable load conditioning. Lastly, the authors have further explored the influence of myocardial layers and fiber orientation on strain imaging and confirmed the previously described pattern of increasing longitudinal deformation as one moves from epicardium to endocardium (Fig. 1). Therefore, as previously demonstrated in adult studies, it remains critical to specify the type of myocardial layer being investigated. Subendocardial longitudinal fibers are most vulnerable to ischemic injury and consequently endocardial GLS remains the predominantly investigated strain metric.

Overall, the authors are to be commended for their important contribution to the understanding of neonatal imaging which should encourage further investigations in this unique patient population. However, there is still much to learn regarding the accuracy of strain-based imaging in neonates regarding temporal strain rate derivatives and regional deformation. There is a critical need to evaluate the ability of neonatal STE to predict long-term clinical sequalae and to evaluate its accuracy in patients with complex structural heart disease and electrophysiologic pathology. 


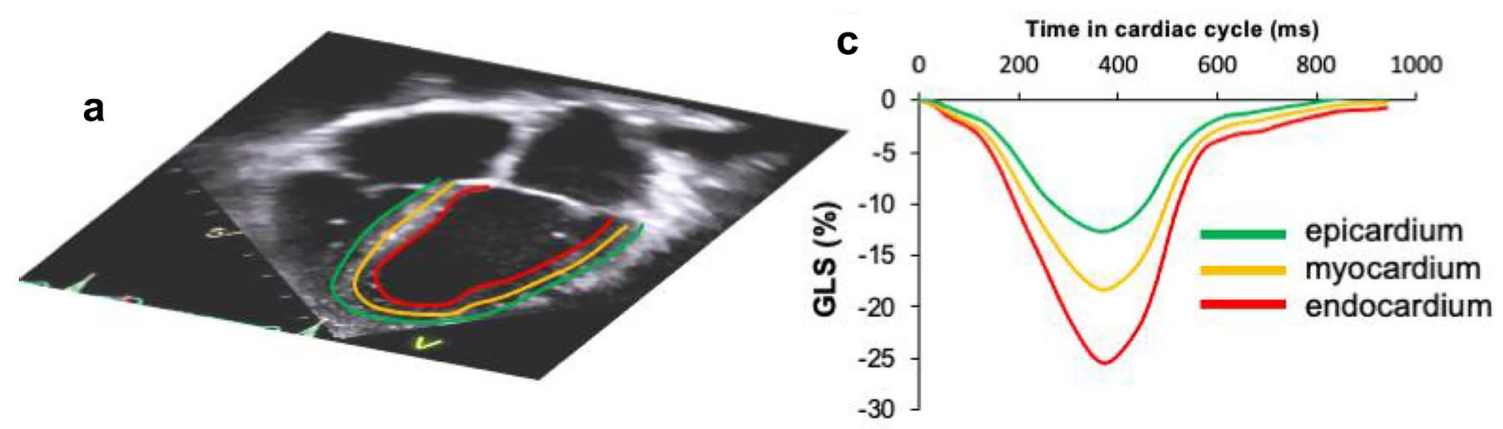

b
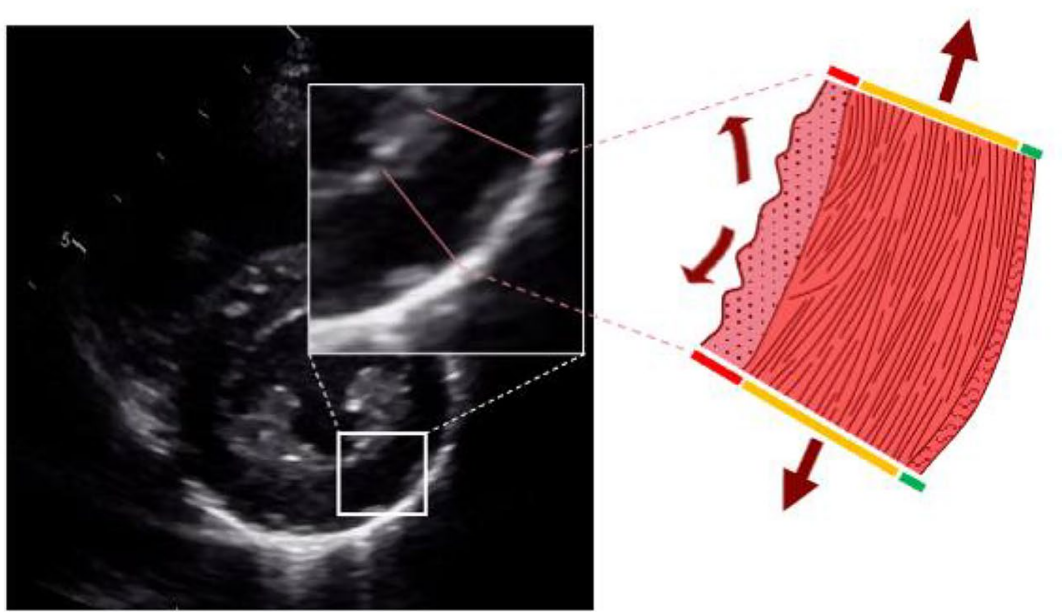

Fig. 1 Myocardial strain evaluation using STE. a GLS evaluated from the apical 4-chamber view can be assessed in specific myocardial layers $\quad$ green $=$ epicardial, yellow $=$ myocardial, red=endocardial $). \quad b$ Para-sternal short axis mid-papillary view provides additional appreciation for different myocardial layers and different fiber orientation within each layer. The inner most subendocardial layer contains lon-

\section{Declarations}

Conflict of interest The authors declare that they have no conflict of interest.

\section{References}

1. Stokke TM, Hasselberg NE, Smedsrud MK et al (2017) Geometry as a confounder when assessing ventricular systolic function: comparison between ejection fraction and strain. J Am Coll Cardiol 70:942-954

gitudinally oriented fibers which are most susceptible to ischemic injury and their deformation evaluated by GLS is frequently associated with clinical outcomes. c GLS waveform for individual myocardial layers throughout the cardiac cycle. Peak endocardial strain typically provides highest values

2. Khan U, Omdal TR, Matre K, Greve G (2021) Speckle tracking derived strain in neonates: planes, layers and drift. Int J Cardiovasc Imaging. https://doi.org/10.1007/s10554-021-02200-8

3. Amzulescu MS, De Craene M, Langet H et al (2019) Myocardial strain imaging: review of general principles, validation, and sources of discrepancies. Eur Hear J Cardiovasc Imaging 20:605-619

Publisher's Note Springer Nature remains neutral with regard to jurisdictional claims in published maps and institutional affiliations. 